\title{
Shifted Euler constants and a generalization of Euler-Stieltjes constants
}

\author{
Tapas Chatterjee ${ }^{1, *}$, Suraj Singh Khurana ${ }^{2}$ \\ Department of Mathematics, Indian Institute of Technology Ropar, Punjab-14001, India
}

\begin{abstract}
The purpose of this article is twofold. First, we introduce the constants $\zeta_{k}(\alpha, r, q)$ where $\alpha \in(0,1)$ and study them along the lines of work done on Euler constant in arithmetic progression $\gamma(r, q)$ by Briggs, Dilcher, Knopfmacher, Lehmer and some other authors. These constants are used for evaluation of certain integrals involving error term for Dirichlet divisor problem with congruence conditions and also to provide a closed form expression for the value of a class of Dirichlet L-series at any real critical point. In the second half of this paper, we consider the behaviour of the Laurent Stieltjes constants $\gamma_{k}(\chi)$ for a principal character $\chi$. In particular we study a generalization of the "Generalized Euler constants" introduced by Diamond and Ford in 2008. We conclude with a short proof for a closed form expression for the first generalized Stieltjes constant $\gamma_{1}(r / q)$ which was given by Blagouchine in 2015.
\end{abstract}

Keywords: Analytic continuation, Dirichlet L-series, Divisor problem, Generalized Euler constants, Riemann Zeta function 2010 MSC: 11M06, 11M99, 11Y60, 11M35, 11K65

\section{Introduction}

It is well known that the Euler's constant $\gamma$ occurs as the constant term in the Laurent series expansion of the Riemann zeta function $\zeta(s)$ at $s=1$. In particular we have

$$
\zeta(s)=\frac{1}{s-1}+\sum_{k=0}^{\infty} \frac{(-1)^{k}}{k !} \gamma_{k}(s-1)^{k}
$$

\footnotetext{
${ }^{*}$ Corresponding author

Email addresses: tapasc@iitrpr.ac.in (Tapas Chatterjee), suraj.khurana@iitrpr.ac.in (Suraj Singh Khurana)

${ }^{1}$ Research of the first author was supported by a NBHM Research Project Grant-in-Aid with grant no. NBHM/2/11/39/2017/R\&D II/3481.

${ }^{2}$ Research of the second author was supported by Council of Scientific and Industrial Research (CSIR), India under File No: 09/1005(0016)/2016-EMR-1.
} 
where $\gamma_{0}$ is the Euler's constant $\gamma$ and in general for $k \geq 0$ the constant $\gamma_{k}$ is known as Euler-Stieltjes constant which is given by the limit

$$
\gamma_{k}:=\lim _{N \rightarrow \infty}\left(\sum_{n=1}^{N} \frac{\log ^{k} n}{n}-\frac{\log ^{k+1} N}{k+1}\right) .
$$

Not only this, the constant $\gamma$ has made its appearance in numerous other works [33] and is therefore considered as fundamental as $\pi$ and $e$. But unlike $\pi$ and $e$, the question of irrationality of $\gamma$ is still open. Consequently, many authors have considered various generalizations of $\gamma$ and studied their properties leading to a vast literature. In Section 2, we briefly review a few generalizations of $\gamma$ important for our discussion and then state the definition of the "Shifted Euler constants" $\zeta_{k}(\alpha, r, q)$ along with the related results. The proofs of these results and some properties of $\zeta_{k}(\alpha, r, q)$ are given in the Section 3. In Section 4, we consider a generalization of "Generalized Euler constants" introduced by Diamond and Ford in [17] in the context of Dirichlet L-series. At the end we give a closed form expression for the first Generalized Stieltjes constant which occurs in the Laurent series expansion of Hurwitz zeta function about the point $s=1$.

\subsection{Notations}

To facilitate our discussion we provide list of the abbreviated notations which will be used throughout this paper. Empty set will be denoted by the symbol $\emptyset$. The value of an empty sum and an empty product will be considered as 0 and 1 respectively. The symbol $\mathbb{N}_{0}$ stands for the set of all non-negative integers. For a complex number $z$, let $\Re(z)$ denote the real part of $z$. The symbol ${ }^{n} P_{i}$ will denote the value $\frac{n !}{(n-i) !}$. The residue of the function $f(z)$ at the point $z=a$ will be written as $\operatorname{Res}_{z=a} f(z)$. The symbol $D_{\alpha}$ will be used to denote the region $\{s \in \mathbb{C}|| s-\alpha|<| \alpha-1 \mid\}$. Notations $\zeta(s), \psi(s)$ and $\zeta(s, x)$ denote respectively the Riemann zeta function, the Digamma function and the Hurwitz zeta function. $F(x, s)$ represents the periodic zeta function and is defined by the series

$$
F(x, s)=\sum_{n=1}^{\infty} \frac{e^{2 \pi i n x}}{n^{s}}
$$

where $\Re(s)>1$ if $x \in \mathbb{Z}$ and $\Re(s)>0$ otherwise. For $r, q \in \mathbb{N}$ where $r \leq q$ the partial zeta function $\zeta(s ; r, q)$ is defined as

$$
\zeta(s ; r, q):=\sum_{\substack{n \geq 1 \\ n \equiv r \bmod q}} \frac{1}{n^{s}}
$$

for $\Re(s)>1$. For a function $f$, the notation $f^{(n)}$ will mean the $n$-th derivative of the function $f$. In particular, $f^{(0)}=f$. For any real number $x,\{x\}$ will represent the fractional part of $x$ and $\lfloor x\rfloor$ will denote the greatest integer less 
than or equal to $x$. Unless otherwise stated the symbol $p_{i}$ will denote the $i$-th prime. The symbol $\mathcal{P}_{r}$ denotes the set consisting of first $r$ many primes. $\overline{\mathbb{Q}}$ represents the field consisting of all algebraic numbers. Let $\alpha \in(0,1), k$ be a non negative integer, $r, q \in \mathbb{N}$ where $r \leq q$. Then the Euler-Stieltjes constant $\gamma_{k}$, the Euler-Lehmer constant $\gamma(r, q)$, the Generalized Euler-Lehmer constant $\gamma_{k}(r, q)$ and the shifted Euler-Lehmer constant $\zeta_{k}(\alpha, r, q)$ are given by (2), (3), (4) and Definition 2.1 respectively.

\section{Shifted Euler constants}

In 1961, Briggs [9] considered the constants $\gamma(r, q)$ associated with arithmetic progressions defined as

$$
\gamma(r, q):=\lim _{x \rightarrow \infty}\left(\sum_{\substack{0<n \leq x \\ n \equiv r \bmod q}} \frac{1}{n}-\frac{1}{q} \log x\right)
$$

where $1 \leq r \leq q$. It is easy to see that $\gamma(1,1)=\gamma$. In [35], using discrete Fourier transforms and some basic tools Lehmer obtained many properties of the constants $\gamma(r, q)$ and derived an elementary proof of the well known Gauss theorem on digamma function $\psi(z)$ at rational arguments. Further the connection of $\gamma(r, q)$ with the class numbers of quadratic fields $\mathbb{Q}(\sqrt{ \pm q})$ and certain infinite series was given in [35]. In particular [35, Theorem 8], it was shown that for a $q$ periodic arithmetic function $f$ satisfying $\sum_{n=1}^{q} f(n)=0$ the Dirichlet series $L(s, f)$ converges at $s=1$ and is given by the following closed form expression

$$
L(1, f)=\sum_{r=1}^{q} f(r) \gamma(r, q) .
$$

The constants $\gamma(r, q)$ are referred to as Euler-Briggs-Lehmer constants or sometimes just Euler-Lehmer constants and results related to their arithmetic nature has been given by Murty and Saradha in [43]. For more results related to arithmetic nature of $\gamma(r, q)$ and its generalization see [21,22]. For the work done on p-adic version of the Euler-Lehmer constants $\gamma(r, q)$ see [12] and [18].

A further generalization of $\gamma(r, q)$ was introduced by Knopfmacher in [32] and later studied in detail by Dilcher in [19]. They considered the generalized Euler-Lehmer constants of higher order which are defined as

$$
\gamma_{k}(r, q):=\lim _{x \rightarrow \infty}\left(\sum_{\substack{n \leq x \\ n \equiv r \bmod q}} \frac{\log ^{k} n}{n}-\frac{\log ^{k+1} x}{q(k+1)}\right) .
$$

It is easy to see that $\gamma_{0}(1,1)=\gamma$ and $\gamma_{0}(r, q)=\gamma(r, q)$ and $\gamma_{k}(1,1)=\gamma_{k}$. Most of the results given in [35] were generalized in [32] using the properties of 
$\gamma_{k}(r, q)$. In Proposition 9 of [32] it was shown that for a $q$ periodic arithmetic function $k$-th derivative of $L(s, f)$ at $s=1$ exists if and only if $\sum_{r=1}^{q} f(r)=0$. Further in the case of existence, the value is given by the following closed form expression

$$
L^{(k)}(1, f)=(-1)^{k} \sum_{r=1}^{q} f(r) \gamma_{k}(r, q) .
$$

For some particular cases where the above identity was used to give explicit expressions the reader may see section 6 of [32].

From the above discussion it seems natural to ask for similar results related to the arithmetic nature or closed form expressions for $L(s, f)$ at points other than $s=1$ as well. We investigate this question for real points lying in the critical strip $0<\Re(s)<1$ which is an important region to study for many Lfunctions. For this we consider a variant of generalized Euler-Lehmer constants and study its properties along the lines of contributions made by Briggs, Dilcher, Knopfmacher, Lehmer and some other authors.

Definition 2.1. For $\alpha \in(0,1), k \in \mathbb{N}_{0}$ and $r, q \in \mathbb{N}$ where $r \leq q$, the Shifted Euler constant $\zeta_{k}(\alpha, r, q)$ is defined as the limit

$$
\zeta_{k}(\alpha, r, q):=\lim _{x \rightarrow \infty}\left(H_{k}(x, \alpha, r, q)-\frac{I_{k}(x, \alpha)}{q}\right)
$$

where

$$
I_{k}(x, \alpha)=\sum_{i=0}^{k}\left((-1)^{i} \frac{{ }^{k} P_{i} \log ^{k-i} x}{(1-\alpha)^{i+1}}\right) x^{1-\alpha}
$$

and

$$
H_{k}(x, \alpha, r, q):=\sum_{\substack{n \leq x \\ n \equiv r \bmod q}} \frac{\log ^{k} n}{n^{\alpha}} .
$$

Here ${ }^{n} P_{i}:=\frac{n !}{(n-i) !}$.

For the existence of the limit $\zeta_{k}(\alpha, r, q)$ see Proposition 3.2. It is easy to see from the definition that $\zeta_{0}(\alpha, 1,1)$ is equal to the value $\zeta(\alpha)[2$, p. 56]. More generally the constants $\zeta_{k}(\alpha, r, q)$ are related with the periodic zeta function $F(x, s)$ which is defined by the series

$$
F(x, s)=\sum_{n=1}^{\infty} \frac{e^{2 \pi i n x}}{n^{s}}
$$

where $\Re(s)>1$ if $x \in \mathbb{Z}$ and $\Re(s)>0$ otherwise. This function has an analytic continuation throughout whole complex plane except when $x \in \mathbb{Z}$ in which case it has a simple pole at $s=1$. The following theorem gives an expression for $\zeta_{k}(\alpha, r, q)$ in terms of derivatives of $F(x, s)$. 
Theorem 2.2. For $\alpha \in(0,1), k \in \mathbb{N}_{0}$ and $b, q \in \mathbb{N}$ such that $b \leq q$ we have the following

$$
\zeta_{k}(\alpha, b, q)=\frac{H_{k}(\alpha)}{q}+\frac{(-1)^{k}}{q} \sum_{a=1}^{q-1} F^{(k)}\left(\frac{a}{q}, \alpha\right) e^{\frac{-2 \pi i a b}{q}}
$$

where

$$
H_{k}(\alpha)=\int_{1}^{\infty}\{t\}\left(\frac{k \log ^{k-1} t-\alpha \log ^{k} t}{t^{1+\alpha}}\right) d t+\frac{(-1)^{k+1} k !}{(1-\alpha)^{k+1}}+\left\lfloor\frac{1}{k+1}\right\rfloor
$$

and $F(x, s)$ is the periodic zeta function.

In the special case when $k=0$, using analytic continuation of $\zeta(s)$ we obtain a series representation for $\zeta_{0}(\alpha, b, q)$.

Corollary 2.3. For $\alpha \neq 1$ and $\Re(\alpha)>0$ we have the following

$$
\zeta_{0}(\alpha, b, q)=\frac{\alpha}{q(\alpha-1)}+\frac{1}{q(1-\alpha)} \sum_{t \geq 1} \sum_{n=2}^{\infty} \frac{(\alpha-1)_{t}}{n^{\alpha+t}}+\frac{1}{q} \sum_{a=1}^{q-1} \sum_{n=1}^{\infty} \frac{e^{\frac{2 \pi i \alpha(n-b)}{q}}}{n^{\alpha}}
$$

where $(s)_{t}=\frac{s(s+1) \cdots(s+t)}{(t+1) !}$ is the classical Pochhammer symbol.

To motivate for the next result we recall that the Laurent series coefficients $\gamma_{k}$ of the function $\zeta(s)$ around the point $s=1$ is well known and has been studied extensively by many authors $[1,8,10,13,14,31,38,58]$. On the contrary, the Laurent series expansion of certain Dirichlet L-functions at points other than the poles seems to be first considered by A. Ivić [28] and was used to evaluate integrals containing error terms related to some well known problems [28, Section 4]. In [36], using the Maclaurin series expansion of $\zeta(s)$ at $s=0$, Lehmer gave an expression for the infinite sum $\sum_{\rho} \frac{1}{\rho^{k}}$ where $\rho$ varies over all the complex zeroes of $\zeta(s)$. For Maclaurin series expansion of Hurwitz zeta function at $s=0$ see [7]. Here we give explicit expressions for the Laurent series expansion of some well known Dirichlet L-functions at points lying on the real line in the critical strip. For this, we make use of the partial zeta function $\zeta(s ; r, q)$ which is defined as

$$
\zeta(s ; r, q):=\sum_{\substack{n \geq 1 \\ n \equiv r \bmod q}} \frac{1}{n^{s}}
$$

for $\Re(s)>1$. Using the well known meromorphic continuation of Hurwitz zeta function $\zeta(s, \alpha)[2$, Chapter 12] and the identity

$$
\zeta(s ; r, q)=q^{-s} \zeta\left(s, \frac{r}{q}\right)
$$


one can easily deduce the meromorphic continuation of $\zeta(s ; r, q)$ over the whole complex plane except at the point $s=1$. Recently Shirasaka gave [53, Theorem(i)] the Laurent series expansion of the function $\zeta(s ; r, q)$ around the point $s=1$ as follows:

$$
\zeta(s ; r, q)=\frac{1}{q(s-1)}+\sum_{k=0}^{\infty} \frac{(-1)^{k}}{k !} \gamma_{k}(r, q)(s-1)^{k} .
$$

It is easy to see that the expansion given by the equation (7) is a generalization of the expansion given in (1). Using (7) and some properties of Hurwitz zeta function Shirasaka derived identities of Lehmer [35], Dilcher [19] and Kanemitsu [30] in a unified manner. We recall that for $\alpha \in(0,1)$ the notation $D_{\alpha}$ represents the region

$$
D_{\alpha}:=\{s \in \mathbb{C}|| s-\alpha|<| \alpha-1 \mid\} .
$$

Theorem 2.4. For $\alpha \in(0,1)$ and $r, q \in \mathbb{N}$ the Taylor series expansion of the function $\zeta(s ; r, q)$ at the point $s=\alpha$ is given by

$$
\zeta(s ; r, q)=\sum_{m=0}^{\infty} \frac{(-1)^{m}}{m !} \zeta_{m}(\alpha, r, q)(s-\alpha)^{m}
$$

and is valid for $s \in D_{\alpha}$.

Some immediate corollaries of the above theorem are the following.

Corollary 2.5. Let $k \geq 0$ and $d$ be a common divisor of $r$ and $q$. Then we have

$$
\zeta_{k}(\alpha, r, q)=\sum_{j=0}^{k}\left(\begin{array}{c}
k \\
j
\end{array}\right) \frac{\log ^{k-j} d}{d^{\alpha}} \zeta_{j}\left(\alpha, \frac{r}{d}, \frac{q}{d}\right) .
$$

Proof. Follows from the equality of the Laurent series expansion of the two functions $\zeta(s ; r, q)$ and $\frac{1}{d^{s}} \zeta\left(s ; \frac{r}{d}, \frac{q}{d}\right)$ at the point $s=\alpha$.

Corollary 2.6. The Taylor series expansion of Hurwitz zeta function $\zeta\left(s, \frac{r}{q}\right)$ around the point $s=\alpha$ is given by

$$
\zeta\left(s, \frac{r}{q}\right)=\sum_{m=0}^{\infty}\left(q^{\alpha} \sum_{j=0}^{m}(-1)^{m-j} \frac{\log ^{j} q}{j !} \frac{\zeta_{m-j}(\alpha, r, q)}{(m-j) !}\right)(s-\alpha)^{m}
$$

and is valid for $s \in D_{\alpha}$.

Proof. Expand $q^{-s}$ at the point $s=\alpha$ in the identity (6) and then use the above theorem. 
In the special case when $r=1$ and $q=1$ we recover from Eq. (8), the expansion of $\zeta(s)$ as given in the equation 2.3 of $[28]^{3}$. Hence, the constants $\zeta_{k}(\alpha, 1,1)$ coincides with $\gamma_{k}\left(s^{\prime}\right)[28$, Equation 2.4] which were used to express integrals involving error terms of Piltz divisor problem [28, Section 4.1]. For work done related to Dirichlet and Piltz divisor problem see [27, Chapter 13] and [56, Chapter 12]. Here we consider a more general Dirichlet divisor problem with congruence conditions $[37,39,42,46,47]$ which is the study of the error term $\Delta_{2}\left(x ; r_{1}, q_{1}, r_{2}, q_{2}\right)$ defined by the equation

$$
\Delta_{2}\left(x ; r_{1}, q_{1}, r_{2}, q_{2}\right)=\sum_{n \leq x} d_{*}(n)-\operatorname{Res}_{s=1}\left(\zeta\left(s ; r_{1}, q_{1}\right) \zeta\left(s ; r_{2}, q_{2}\right) \frac{x^{s}}{s}\right)
$$

where $d_{*}(n)$ is the number of elements in the set

$$
\left\{\left(n_{1}, n_{2}\right) \in \mathbb{N}^{2}: n=n_{1} n_{2}, n_{1} \equiv r_{1} \bmod q_{1}, n_{2} \equiv r_{2} \bmod q_{2}\right\} .
$$

It is known from Richert's work [51] and Huxley's estimates [24] that

$$
\Delta_{2}\left(q_{1} q_{2} x ; r_{1}, q_{1}, r_{2}, q_{2}\right) \ll x^{\frac{131}{416}}(\log x)^{\frac{26947}{8320}} .
$$

Here we express a family of integrals involving the error term $\Delta_{2}\left(x ; r_{1}, q_{1}, r_{2}, q_{2}\right)$ similarly to the results obtained for $\Delta_{k}[20,34,54]$ in the case of classical Dirichlet divisor problem.

Theorem 2.7. Let $\tilde{\Delta}_{2}(x)$ denote the error term $\Delta_{2}\left(x ; r_{1}, q_{1}, r_{2}, q_{2}\right)$ given by (9). Then for any non negative integer $k$ we have

$$
\begin{aligned}
\int_{1}^{\infty} & \frac{\log ^{k} x-k \log ^{k-1} x}{x^{2}} \tilde{\Delta}_{2}(x) d x \\
= & \sum_{i=0}^{k}\left(\begin{array}{c}
k \\
i
\end{array}\right) \gamma_{k-i}\left(r_{2}, q_{2}\right) \gamma_{i}\left(r_{1}, q_{1}\right)+\left\lfloor\frac{1}{k+1}\right\rfloor\left(\frac{1}{q_{1} q_{2}}-\frac{\gamma_{0}\left(r_{1}, q_{1}\right)}{q_{2}}-\frac{\gamma_{0}\left(r_{2}, q_{2}\right)}{q_{1}}\right) \\
& \quad-\frac{\gamma_{k+1}\left(r_{2}, q_{2}\right)}{q_{1}(k+1)}-\frac{\gamma_{k+1}\left(r_{1}, q_{1}\right)}{q_{2}(k+1)}
\end{aligned}
$$

and for $\alpha \in\left(\frac{131}{416}, 1\right)$ we have

$$
\begin{aligned}
\int_{1}^{\infty} & \frac{\alpha \log ^{k} x-k \log ^{k-1} x}{x^{1+\alpha}} \tilde{\Delta}_{2}(x) d x \\
& =\sum_{l=0}^{k} \zeta_{l}\left(\alpha, r_{1}, q_{1}\right) \zeta_{k-l}\left(\alpha, r_{2}, q_{2}\right)+\left\lfloor\frac{1}{k+1}\right\rfloor\left(\frac{1}{q_{1} q_{2}}-\frac{\gamma_{0}\left(r_{1}, q_{1}\right)}{q_{2}}-\frac{\gamma_{0}\left(r_{2}, q_{2}\right)}{q_{1}}\right) \\
& -\frac{1}{q_{1} q_{2}}\left(\frac{(k+1) !}{(\alpha-1)^{k+2}}\right)-\left(\frac{\gamma_{0}\left(r_{1}, q_{1}\right)}{q_{2}}+\frac{\gamma_{0}\left(r_{2}, q_{2}\right)}{q_{1}}\right)\left(\frac{k !}{(\alpha-1)^{k+1}}\right) .
\end{aligned}
$$

\footnotetext{
${ }^{3}$ There seems to be a missing term $\frac{k !}{\left(s^{\prime}-1\right)^{k+1}}$ in the expression 2.4 for $\gamma_{k}\left(s^{\prime}\right)$
} 
In the special case when $k=0$ and $r_{1}=q_{1}=r_{2}=q_{2}=1$ the identity (11) reduces to the one given by Lavrik, Israilov and Ëdgorov in [34] ${ }^{4}$. We remark here that by using the idea of Sitaramachandra Rao in [54], the proof of Theorem 2.7 can be generalized for the general error term $\tilde{\Delta}_{k}$.

Now we discuss the next consequence of Theorem 2.4 in connection with the Dirichlet series with periodic coefficients.For a $q$ periodic arithmetic function $f$, the Laurent series expansion of $L(s, f)$ at the point $s=1$ was given by Ishibashi and Kanemitsu in [25]. They showed that [25, Theorem 2] the Laurent(or Taylor) series expansion of $L(s, f)$ at $s=1$ is given by

$$
L(s, f)=\frac{\gamma_{-1}(f)}{s-1}+\sum_{n=1}^{\infty} \frac{\gamma_{n}(f)}{n !}(s-1)^{n}
$$

where a closed form expression of $\gamma_{i}$ is explicitly given for $i=-1,0,1,2$. Such an expansion was used to study problems related to the product of $\mathrm{L}$ functions [25, Section 2]. We state the following result regarding the Laurent series expansion for $L(s, f)$ which follows directly from the identity

$$
L(s, f)=\sum_{r=1}^{q} f(r) \zeta(s ; r, q)
$$

and Theorem 2.4.

Theorem 2.8. For $\alpha \in(0,1)$ and a $q$ periodic arithmetic function $f$, the Dirichlet $L$ function $L(s, f)$ has the following Taylor series expansion at the point $s=\alpha$

$$
L(s, f)=\sum_{k=0}^{\infty} \frac{(-1)^{k}}{k !}\left(\sum_{r=1}^{q} f(r) \zeta_{k}(\alpha, r, q)\right)(s-\alpha)^{k}
$$

which is valid for $s \in D_{\alpha}$. Moreover, the $k$-th derivative of $L(s, f)$ at $s=\alpha$ has the following closed form expression

$$
L^{(k)}(\alpha, f)=(-1)^{k} \sum_{r=1}^{q} f(r) \zeta_{k}(\alpha, r, q) .
$$

An infinite series expression for the constants $\zeta_{k}(\alpha, r, q)$ can be given as follows.

Corollary 2.9. For any a such that $(a, q)=1$ and $1 \leq a \leq q$ we have

$$
\zeta_{m}(\alpha, a, q)=\frac{m !}{q(\alpha-1)^{m+1}}+\frac{1}{\phi(q)} \sum_{k \geq m} \sum_{\chi} \frac{(-1)^{k} \bar{\chi}(a) \gamma_{k}(\chi)}{(k-m) !}(1-\alpha)^{k-m}
$$

where $\chi$ varies over all the Dirichlet characters modulo $q$.

\footnotetext{
${ }^{4}$ The authors in [34] use a slightly different definition for the constant $\gamma_{k}$.
} 
Proof. Using (13) and (14) we can obtain two expressions for $L^{(m)}(\alpha, \chi)$. Since they must be equal we get

$$
\sum_{r=1}^{q} \chi(r) \zeta_{m}(\alpha, r, q)=\frac{\gamma_{-1}(\chi) m !}{(\alpha-1)^{m+1}}+\sum_{k \geq m} \frac{(-1)^{k} \gamma_{k}(\chi)}{(k-m) !}(1-\alpha)^{k-m} .
$$

Now use the well known orthogonality relation of Dirichlet characters $\chi$ to deduce the result.

The value of $L(s, \chi)$ and its derivatives at the point $s=\frac{1}{2}$ is of special interest to many authors $[3,11,29,40,55]$. It is conjectured that $L\left(\frac{1}{2}, \chi\right) \neq 0$ for all primitive Dirichlet characters $\chi$. Recently Murty and Tanabe [44] studied the relation between the arithmetic nature of $e^{\gamma}$ and non vanishing of central values of Artin L functions. From Theorem 2.8 we can observe the following condition.

Corollary 2.10. For any $k$ if the set $\left\{\zeta_{k}\left(\frac{1}{2}, r, q\right) \mid 1 \leq r \leq q\right\}$ is linearly independent over $\overline{\mathbb{Q}}$ then $L^{(k)}\left(\frac{1}{2}, \chi\right) \neq 0$ for all Dirichlet character $\chi$ modulo $q$.

Proof. From the hypothesis it is clear that 0 does not belong to the given set. Now suppose on the contrary that $L^{(k)}\left(\frac{1}{2}, \chi\right)=0$. Then from (15) it follows that when $\alpha=1 / 2$ and $f=\chi$, there exists a non trivial linear combination from the set $\left\{\zeta_{k}\left(\frac{1}{2}, r, q\right) \mid 1 \leq r \leq q\right\}$ over $\overline{\mathbb{Q}}$ which is equal to 0 . This contradicts the linear independence of the given set.

\section{Proof of the results in Section 2}

Lemma 3.1. For a non negative integer $k$ and $\alpha \in(0,1)$ we have

$$
\sum_{n \leq x} \frac{\log ^{k} n}{n^{\alpha}}=I_{k}(x, \alpha)+H_{k}(\alpha)+O\left(\frac{\log ^{k} x}{x^{\alpha}}\right)
$$

where

$$
H_{k}(\alpha)=\int_{1}^{\infty}\{t\}\left(\frac{k \log ^{k-1} t-\alpha \log ^{k} t}{t^{1+\alpha}}\right) d t+\frac{(-1)^{k+1} k !}{(1-\alpha)^{k+1}}+\left\lfloor\frac{1}{k+1}\right\rfloor
$$

and

$$
I_{k}(x, \alpha)=\sum_{i=0}^{k}\left(\frac{{ }^{k} P_{i}(-1)^{i} \log ^{k-i} x}{(1-\alpha)^{i+1}}\right) x^{1-\alpha} .
$$

Proof. Apply Abel summation formula [2, Theorem 4.2] and use induction on $k$.

Proposition 3.2. The limit defined as $\zeta_{k}(\alpha, r, q)$ exists for $\alpha \in(0,1)$. 
Proof. Consider the partial sum

$$
A(x)=\sum_{\substack{n \leq x \\ n \equiv r \bmod q}} 1
$$

Then we have

$$
\begin{aligned}
H_{k}(x, \alpha, r, q) & =\sum_{\substack{n \leq x \\
n \equiv r \bmod q}} \frac{\log ^{k} n}{n^{\alpha}}=\int_{1^{-}}^{x} \frac{\log ^{k} t}{t^{\alpha}} d A(t) \\
& =\int_{1^{-}}^{x} \frac{\log ^{k} t}{t^{\alpha}} d\left(\left(\frac{t-r}{q}\right)-\left\{\frac{t-r}{q}\right\}\right) \\
& =\frac{1}{q} \int_{1}^{x} \frac{\log ^{k} t}{t^{\alpha}} d t+C+O\left(\frac{\log ^{k} x}{x^{\alpha}}\right)
\end{aligned}
$$

for some constant C. Now the result follows from Lemma 3.1

Corollary 3.3. $\zeta_{k}(\alpha, r, q)$ satisfy the following properties :

1. $\zeta_{0}(\alpha, 0,1)=H_{0}(\alpha)=\zeta(\alpha)$

2. $\zeta_{k}(\alpha, r \pm q, q)=\zeta_{k}(\alpha, r, q)$

3. $\sum_{r=1}^{q} \zeta_{k}(\alpha, r, q)=H_{k}(\alpha)$

4. $\sum_{j=0}^{q-1} \zeta_{k}(\alpha, r+j m, m q)=\zeta_{k}(\alpha, r, m)$.

Proof. Part 1, 2 and 3 follow immediately from the definition of $\zeta_{k}(\alpha, r, q)$. For the part 4 notice that

$$
\sum_{j=0}^{q-1} \zeta_{k}(\alpha, r+j m, m q)=\sum_{j=0}^{q-1} \lim _{x \rightarrow \infty}\left(\sum_{\substack{0<n \leq x \\ n \equiv r+j m \bmod m q}} \frac{\log ^{k} n}{n^{\alpha}}-\frac{I_{k}(x, \alpha)}{m q}\right) .
$$

Here in the right hand side $n=(r+j m)+n^{\prime} m q$ where $n^{\prime} \geq 0$ and $j \in$ $\{0,1,2, \cdots, q-1\}$. This means $n=r+\left(n^{\prime} q+j\right) m$ where $n^{\prime} q+j$ varies over all non-negative integers. Hence

$$
\sum_{j=0}^{q-1} \zeta_{k}(\alpha, r+j m, m q)=\lim _{x \rightarrow \infty}\left(\sum_{\substack{0<n \leq x \\ 0 \equiv r \bmod m}} \frac{\log ^{k} n}{n^{\alpha}}-\frac{I_{k}(x, \alpha)}{m}\right)=\zeta_{k}(\alpha, r, m) .
$$




\subsection{Proof of Theorem 2.2}

Consider the discrete Fourier transform $f$ defined by

$$
f(a):=\sum_{b=0}^{q-1} \zeta_{k}(\alpha, b, q) e^{\frac{2 \pi i a b}{q}} .
$$

Now for $a=0$ we have

$$
f(0)=\sum_{b=0}^{q-1} \zeta_{k}(\alpha, b, q)=H_{k}(\alpha) .
$$

On the other hand for $a \neq 0$, we have

$$
\begin{aligned}
f(a) & =\sum_{b=0}^{q-1} \lim _{x \rightarrow \infty}\left(H_{k}(x, \alpha, b, q)-\frac{I_{k}(x, \alpha)}{q}\right) e^{\frac{2 \pi i a b}{q}} \\
& =\lim _{x \rightarrow \infty}\left(\sum_{b=0}^{q-1} H_{k}(x, \alpha, b, q) e^{\frac{2 \pi i a b}{q}}-\frac{I_{k}(x, \alpha)}{q} \sum_{b=0}^{q-1} e^{\frac{2 \pi i a b}{q}}\right) \\
& =\lim _{x \rightarrow \infty}\left(\sum_{b=0}^{q-1}\left(\sum_{\substack{0<n \leq x \\
n \equiv b \bmod q}} \frac{e^{\frac{2 \pi i a n}{q}} \log ^{k} n}{n^{\alpha}}\right)\right) \\
& =\sum_{n=1}^{\infty} \frac{e^{\frac{2 \pi i a n}{q}} \log ^{k} n}{n^{\alpha}} .
\end{aligned}
$$

Hence we get

$$
F^{(k)}\left(\frac{a}{q}, \alpha\right)=(-1)^{k} \sum_{b=0}^{q-1} \zeta_{k}(\alpha, b, q) e^{\frac{2 \pi i a b}{q}} .
$$

Applying Fourier inversion on the above identity gives the desired result.

\subsection{Proof of Corollary 2.3}

We know that

$$
\begin{aligned}
\zeta_{0}(\alpha, b, q) & =\frac{H_{0}(\alpha)}{q}+\sum_{a=1}^{q-1} F\left(\frac{a}{q}, \alpha\right) e^{\frac{-2 \pi i a b}{q}} \\
& =\frac{\zeta(\alpha)}{q}+\sum_{a=1}^{q-1} F\left(\frac{a}{q}, \alpha\right) e^{\frac{-2 \pi i a b}{q}} .
\end{aligned}
$$

Now we shall use the following identity given by Ramanujan in [48]

$$
1=\sum_{t \geq 0}(s-1)_{t}(\zeta(s+t)-1) .
$$


From this one can deduce the analytic continuation for $\zeta(s)$

$$
\zeta(s)=\frac{s}{s-1}-\frac{1}{s-1} \sum_{t \geq 1}(s-1)_{t}(\zeta(s+t)-1) .
$$

For $s=\alpha$ where $\Re(\alpha)>0$ it follows from Ramanujan's identity that

$$
\begin{aligned}
\zeta_{0}(\alpha, b, q) & =\frac{\alpha}{q(\alpha-1)}+\frac{1}{q(1-\alpha)} \sum_{t \geq 1}(\alpha-1)_{t}(\zeta(\alpha+t)-1)+\frac{1}{q} \sum_{a=1}^{q-1} \sum_{n=1}^{\infty} \frac{e^{\frac{2 \pi i n a}{q}}}{n^{\alpha}} e^{\frac{-2 \pi i a b}{q}} \\
& =\frac{\alpha}{q(\alpha-1)}+\frac{1}{q(1-\alpha)} \sum_{t \geq 1}(\alpha-1)_{t}\left(\sum_{n=2}^{\infty} \frac{1}{n^{\alpha+t}}\right)+\frac{1}{q} \sum_{a=1}^{q-1} \sum_{n=1}^{\infty} \frac{e^{\frac{2 \pi i n a}{q}}}{n^{\alpha}} e^{\frac{-2 \pi i a b}{q}} \\
& =\frac{\alpha}{q(\alpha-1)}+\frac{1}{q(1-\alpha)} \sum_{t \geq 1} \sum_{n=2}^{\infty} \frac{(\alpha-1)_{t}}{n^{\alpha+t}}+\frac{1}{q} \sum_{a=1}^{q-1} \sum_{n=1}^{\infty} \frac{e^{\frac{2 \pi i a(n-b)}{q}}}{n^{\alpha}} .
\end{aligned}
$$

This completes the proof.

For a general $k$, since we have analytic continuation of the periodic zeta function $F(x, s)$ we can deduce the following translational formula.

Proposition 3.4. For $l, b, q \in \mathbb{N}$ where $b \leq q$ the function $\zeta_{k}(\alpha, b, q)$ obtained by meromorphic continuation of the periodic zeta function $F(x, s)$ satisfies the following recursive equation

$$
\zeta_{k+l}(\alpha, b, q)=\frac{H_{k+l}(\alpha)}{q}+(-1)^{l}\left(\zeta_{k}^{(l)}(\alpha, b, q)-\frac{H_{k}^{l}(\alpha)}{q}\right)
$$

Proof. Take derivative of equation (5) $l$ times and compare it with (5).

\subsection{Proof of Theorem 2.4}

Denote the partial sum

$$
A(x):=\sum_{\substack{n \leq x \\ n \equiv r \bmod q}} 1=\left\lfloor\frac{x-r}{q}\right\rfloor .
$$

Then for $\Re(s)>1$, by the use of Riemann Stieltjes integral we have

$$
\begin{aligned}
\zeta(s ; r, q) & =\int_{1}^{\infty} \frac{d A(t)}{t^{s}}=\frac{1}{q} \int_{1}^{\infty} \frac{1}{t^{s}}+\int_{1}^{\infty} \frac{1}{t^{s}} d\left(\left\lfloor\frac{t-r}{q}\right\rfloor-\left(\frac{t-r}{q}\right)\right) \\
& =\frac{1}{q(s-1)}+\int_{1}^{\infty} \frac{1}{t^{s}} d\left(\left\lfloor\frac{t-r}{q}\right\rfloor-\left(\frac{t-r}{q}\right)\right) .
\end{aligned}
$$


The above sum is an analytic function in the region $\Re(s)>0$ except for a simple pole at $s=1$. Hence we have the analytic continuation of $\zeta(s ; r, q)$ to this region. Now expand both the function $\frac{1}{s-1}$ and $\frac{1}{t^{s}}$ as a Taylor series around the point $s=\alpha$. We get

$$
\begin{aligned}
\zeta(s ; r, q)= & \frac{1}{q}\left(\sum_{m=0}^{\infty} \frac{(-1)^{m}}{(\alpha-1)^{m+1}}(s-\alpha)^{m}\right) \\
& \left.+\int_{1}^{\infty}\left(\frac{(-1)^{m}}{m !} \frac{\log ^{m} t}{t^{\alpha}}(s-\alpha)^{m}\right) d\left(\mid \frac{t-r}{q}\right\rfloor-\left(\frac{t-r}{q}\right)\right) \\
= & \frac{1}{q}\left(\sum_{m=0}^{\infty} \frac{(-1)^{m}}{(\alpha-1)^{m+1}}(s-\alpha)^{m}\right) \\
& +\sum_{m=0}^{\infty} \frac{(-1)^{m}}{m !}(s-\alpha)^{m} \lim _{N \rightarrow \infty}\left(\sum_{\substack{n \leq N \\
n \equiv r \bmod q}} \frac{\log ^{m} n}{n^{\alpha}}-\frac{1}{q} \int_{1}^{N} \frac{\log ^{m} t}{t^{\alpha}} d t\right) .
\end{aligned}
$$

Using Proposition 3.2 we get

$$
\begin{aligned}
\zeta(s ; r, q)= & \frac{1}{q}\left(\sum_{m=0}^{\infty} \frac{(-1)^{m}}{(\alpha-1)^{m+1}}(s-\alpha)^{m}\right) \\
& +\sum_{m=0}^{\infty} \frac{(-1)^{m}}{m !}(s-\alpha)^{m}\left(\zeta_{m}(\alpha, r, q)+\frac{(-1)^{m} m !}{q(1-\alpha)^{m+1}}\right) .
\end{aligned}
$$

Simplifying the above sum gives the desired result.

\subsection{Proof of Theorem 2.7}

For $\Re(s)>1$ we have

$$
\zeta\left(s ; r_{1}, q_{1}\right) \zeta\left(s ; r_{2}, q_{2}\right)=L\left(s, d_{*}\right)=\sum_{n=1}^{\infty} \frac{d_{*}(n)}{n^{s}}=\int_{1^{-}}^{\infty} x^{-s} d A(x)
$$

where $A(x)$ is given by

$$
A(x)=\sum_{n \leq x}^{\prime} d_{*}(n)=\Delta_{*}\left(x ; r_{1}, q_{1}, r_{2}, q_{2}\right)+x P_{k-1}\left(\log x, r_{1}, q_{1}, r_{2}, q_{2}\right) .
$$

By the use of Perron's inversion formula [27, Appendix A.3] the main term can be written as

$$
x P_{2}\left(\log x, r_{1}, q_{1}, r_{2}, q_{2}\right)=\operatorname{Res}_{s=1}\left(\zeta\left(s ; r_{1}, q_{1}\right) \zeta\left(s ; r_{2}, q_{2}\right) \frac{x^{s}}{s}\right) .
$$


Further with the help of the Laurent series expansion $(7)$ of $\zeta(s ; r, q)$ at $s=1$ we get

$$
P_{2}\left(\log x, r_{1}, q_{1}, r_{2}, q_{2}\right)=\frac{\log x}{q_{1} q_{2}}+\frac{\gamma_{0}\left(r_{1}, q_{1}\right)}{q_{2}}+\frac{\gamma_{0}\left(r_{2}, q_{2}\right)}{q_{1}}-\frac{1}{q_{1} q_{2}} .
$$

Using (17) we can write down the integral in (16) as the sum of $S_{1}(s)$ and $S_{2}(s)$ where

$$
\begin{aligned}
S_{1}(s) & =\int_{1^{-}}^{\infty} x^{-s} d\left(x P_{2}\left(\log x, r_{1}, q_{1}, r_{2}, q_{2}\right)\right) \\
& =\frac{1}{q_{1} q_{2}}\left(\frac{1}{(s-1)^{2}}\right)+\left(\frac{\gamma_{0}\left(r_{1}, q_{1}\right)}{q_{2}}+\frac{\gamma_{0}\left(r_{2}, q_{2}\right)}{q_{1}}\right)\left(\frac{1}{s-1}\right) .
\end{aligned}
$$

and

$$
S_{2}(s)=\int_{1^{-}}^{\infty} x^{-s} d \Delta_{*}\left(x ; r_{1}, q_{1}, r_{2}, q_{2}\right)=-\Delta_{*}(1)+s \int_{1}^{\infty} \frac{\Delta_{*}\left(x ; r_{1}, q_{1}, r_{2}, q_{2}\right)}{x^{s+1}} d x .
$$

It follows from (10) and the above expressions for $S_{1}(s)$ and $S_{2}(s)$ that the Dirichlet L-series $L\left(s, d_{*}\right)$ can be analytically continued to the half plane $\Re(s)>$ $\frac{131}{416}$ except for the pole of order 2 at $s=1$. Using the Taylor series expansion of the function $S_{2}(s)$ about the point $s=1$ one can deduce the Laurent series expansion for $L\left(s, d_{*}\right)$ at $s=1$ as follows

$$
\begin{aligned}
L\left(s, d_{*}\right)= & \frac{1}{q_{1} q_{2}}\left(\frac{1}{(s-1)^{2}}\right)+\left(\frac{\gamma_{0}\left(r_{1}, q_{1}\right)}{q_{2}}+\frac{\gamma_{0}\left(r_{2}, q_{2}\right)}{q_{1}}\right)\left(\frac{1}{s-1}\right) \\
& +\sum_{k=0}^{\infty}\left(\frac{(-1)^{k}}{k !} \int_{1^{-}}^{\infty} \frac{\log ^{k} x}{x} d \Delta_{*}\left(x, r_{1}, q_{1}, r_{2}, q_{2}\right)\right)(s-1)^{k} .
\end{aligned}
$$

To obtain (11) simply equate the coefficient of $(s-1)^{k}$ in the above Laurent series expansion of $L\left(s, d_{*}\right)$ to the coefficient of $(s-1)^{k}$ in the expansion of the product $\zeta\left(s ; r_{1}, q_{1}\right) \zeta\left(s ; r_{2}, q_{2}\right)$ by using (7). Similarly to get (12) expand the functions $S_{1}(s)$ and $S_{2}(s)$ around the point $s=\alpha$ to get a Laurent series expansion for $L\left(s, d_{*}\right)$ at $s=\alpha$ and equate it to the expansion coming from Theorem 2.4. This completes the proof.

\section{A generalization of Euler-Stieltjes constant}

The Laurent series expansion (13) when $f$ is a Dirichlet character $\chi$ modulo $q$ becomes

$$
L(s, \chi)=\frac{\gamma_{-1}(\chi)}{s-1}+\sum_{k=1}^{\infty} \frac{(-1)^{k} \gamma_{k}(\chi)}{k !}(s-1)^{k}
$$


where $\gamma_{k}(\chi)=\sum_{r=1}^{q} \chi(r) \gamma_{k}(r, q)[32]$ for $k \geq 0$ and $\gamma_{-1}(\chi)=\frac{\phi(q)}{q}$ if $\chi$ is principal character $\chi_{0}$ and 0 otherwise. For a non principal character $\chi$, closed form expressions and explicit upper bounds for the constants $\gamma_{k}(\chi)$ have been studied by many authors $[16,23,26,30,52,57]$. For the principal character $\chi_{0}$ modulo $q$ the expression for the constant $\gamma_{0}\left(\chi_{0}\right)$ was given by Redmond in 1982 [49, Lemma 4] (see also [50]). The general expression for $\gamma_{k}\left(\chi_{0}\right)$ was given by Shirasaka [53, Proposition 7]. He proved that

$$
\gamma_{k}\left(\chi_{0}\right)=\sum_{\substack{r=1 \\(r, q)=1}}^{q} \gamma_{k}(r, q)
$$

and

$$
\gamma_{k}\left(\chi_{0}\right)=\sum_{j=0}^{k}\left(\begin{array}{l}
k \\
j
\end{array}\right) \gamma_{j} N_{k-j}(q)-\frac{1}{k+1} N_{k+1}(q)
$$

where

$$
N_{k}(q):=q \sum_{d \mid q} \frac{\mu(d) \log ^{k} d}{d} .
$$

Observing from the definition (4) of $\gamma_{k}(r, q)$ and (18) we state an aysmptotic representation of $\gamma_{k}\left(\chi_{0}\right)$ as follows.

Proposition 4.1. For $k \geq 0$ and the Dirichlet character $\chi_{0}$ modulo $q$, the Laurent series expansion at $s=1$ is given by

$$
L\left(s, \chi_{0}\right)=\frac{\phi(q)}{q(s-1)}+\sum_{k=0}^{\infty} \frac{(-1)^{k} \gamma_{k}\left(\chi_{0}\right)}{k !}(s-1)^{k}
$$

where

$$
\gamma_{k}\left(\chi_{0}\right)=\lim _{x \rightarrow \infty}\left(\sum_{\substack{n \leq x \\(n, \operatorname{rad}(q))=1}} \frac{\log ^{k} n}{n}-\prod_{p \mid \operatorname{rad}(q)}\left(1-\frac{1}{p}\right) \frac{\log ^{k+1} x}{k+1}\right)
$$

and $\operatorname{rad}(n)$ denotes the radical of $n$.

In the case when $k=0$ and $q=1$, the expression (20) reduces to the asymptotic representation (2) of $\gamma_{k}$ which was first discovered by Stieltjes and then later by many other authors [6, p. 538]. In 2008, Diamond and Ford [17] considered the constants $\gamma(\wp)$ associated to a finite set of primes $\wp$ as follows:

$$
\gamma(\wp):=\lim _{x \rightarrow \infty}\left(\sum_{\substack{n \leq x \\\left(n, \prod_{p \in \wp} p\right)=1}} \frac{1}{n}-\delta_{\wp} \log x\right)
$$


where $\delta_{\wp}=\prod_{p \in \wp}\left(1-\frac{1}{p}\right)$. It is easy to see that $\gamma(\emptyset)=\gamma$. A simple observation shows that

$$
\gamma(\wp)=\lim _{s \rightarrow 1}\left[\zeta(s) \prod_{p \in \wp}\left(1-\frac{1}{p^{s}}\right)-\frac{\delta_{\wp}}{s-1}\right]=\gamma_{0}\left(\chi_{0}\right)
$$

where $\chi_{0}$ is the principal Dirichlet character modulo $q=\prod_{p \in \wp} p$. Hence Proposition 1 in [17] and Lemma 2 in [45] are a restatement of Lemma 4 in [49]. However, the constants $\gamma(\wp)$ are of special importance when $\wp=\mathcal{P}_{r}$ where $\mathcal{P}_{r}$ is the set of first $r$ primes. This is mainly because Diamond and Ford [17, Corollary 1] proved that the Riemann Hypothesis is true if and only if " $\gamma\left(\mathcal{P}_{r}\right)>e^{-\gamma}$ for all $r \geq 0$ ". Here we consider the behaviour of the constants $\gamma_{k}\left(\mathcal{P}_{r}\right):=\gamma_{k}\left(\chi_{0}\right)$ where $\chi_{0}$ is the principal Dirichlet character modulo $q=\prod_{p \in \mathcal{P}_{r}} p$. For further discussion we need an expression for $\gamma_{k}\left(\mathcal{P}_{r}\right)$ in terms of generalized von Mangoldt function $\Lambda_{m}:=\sum_{d \mid n} \mu(d) \log ^{k}\left(\frac{n}{d}\right)$.

Proposition 4.2. For a finite set of primes $S$ denote $P_{S}=\prod_{p \in S} p$. Then we have

$$
\gamma_{k}\left(\mathcal{P}_{r}\right)=\prod_{p \mid P_{r}}\left(1-\frac{1}{p}\right)\left(\sum_{i=0}^{k}\left(\sum_{S \subseteq \mathcal{P}_{r}} \frac{L_{k-i}\left(P_{S}\right)}{\prod_{p \mid P_{S}}(p-1)}\right)\left(\begin{array}{c}
k \\
i
\end{array}\right) \gamma_{i}-\frac{1}{k+1} \sum_{S \subseteq \mathcal{P}_{r}} \frac{L_{k+1}\left(P_{S}\right)}{\prod_{p \mid P_{S}}(p-1)}\right) .
$$

where

$$
L_{k}:=\sum_{m=0}^{k}\left(\begin{array}{c}
k \\
m
\end{array}\right)(-1)^{m} \log ^{k-m} \Lambda_{m}
$$

and $P_{r}:=\prod_{p \in \mathcal{P}_{r}} p$.

Proof. Firstly observe that

$$
\begin{aligned}
\sum_{d \mid n} \mu(d) \log ^{k}(d) & =\sum_{d \mid n} \mu(d)\left(\log (n)-\log \left(\frac{n}{d}\right)\right)^{k} \\
& =\sum_{m=0}^{k}\left(\begin{array}{c}
k \\
m
\end{array}\right)(-1)^{m} \log ^{k-m}(n) \Lambda_{m}(n) .
\end{aligned}
$$


With the help of Mobius inversion we can express $\frac{N_{k}\left(P_{r}\right)}{q}$ in the following way:

$$
\begin{aligned}
\frac{N_{k}\left(P_{r}\right)}{q} & =\sum_{d \mid P_{r}} \frac{\mu(d) \log ^{k}(d)}{d}=\sum_{d \mid P_{r}} \frac{\sum_{d_{1} \mid d} L_{k}\left(d_{1}\right) \mu\left(\frac{d}{d_{1}}\right)}{d}=\sum_{d_{1} d_{2} \mid P_{r}} \frac{L_{k}\left(d_{1}\right) \mu\left(d_{2}\right)}{d_{1} d_{2}} \\
& =\sum_{d_{1} \mid P_{r}} \frac{L_{k}\left(d_{1}\right)}{d_{1}} \sum_{d_{2} \mid P_{r}} \frac{\mu\left(d_{2}\right)}{d_{2}}=\sum_{d_{1} \mid P_{r}} \frac{L_{k}\left(d_{1}\right)}{d_{1}} \sum_{d_{2} \mid P_{r}} \frac{\mu\left(d_{2}\right)}{d_{1}} \\
& =\sum_{S \subseteq \mathcal{P}_{r}} \frac{L_{k}\left(P_{S}\right)}{P_{S}} \prod_{p \mid \frac{P_{r}}{P_{S}}}\left(1-\frac{1}{p}\right) \prod_{p \mid P_{S}}\left(1-\frac{1}{p}\right) \prod_{p \mid P_{S}}\left(1-\frac{1}{p}\right)^{-1} \\
& =\sum_{S \subseteq \mathcal{P}_{r}} \frac{L_{k}\left(P_{S}\right)}{P_{S}} \prod_{p \mid P_{r}}\left(1-\frac{1}{p}\right) \prod_{p \mid P_{S}}\left(1-\frac{1}{p}\right)^{-1} \\
& =\prod_{p \mid P_{r}}\left(1-\frac{1}{p}\right) \sum_{S \subseteq \mathcal{P}_{r}} \frac{L_{k}\left(P_{S}\right)}{\prod_{p \mid P_{S}}(p-1)} .
\end{aligned}
$$

Now use the above expression along with (19) to complete the proof.

For $r=0$ it is already known from a result of Briggs [8, Theorem 1] that for $k \geq 0$, the constant $\gamma_{k}\left(\mathcal{P}_{0}\right)$ changes sign infinitely often. This was further improved by Mitrović [41, Theorem 4]. On the other hand, for a general $r \geq 0$ and $k=0$ it was shown [17, Theorem 2] that there are infinitely many integers $r$ for which $\gamma_{0}\left(\mathcal{P}_{r+1}\right)>\gamma_{0}\left(\mathcal{P}_{r}\right)$ and infinitely many integers $r$ for which $\gamma_{0}\left(\mathcal{P}_{r+1}\right)<$ $\gamma_{0}\left(\mathcal{P}_{r}\right)$. More generally using Proposition 4.2 one can give a relation between the two consecutive constants $\gamma_{k}\left(\mathcal{P}_{r}\right)$ and $\gamma_{k}\left(\mathcal{P}_{r+1}\right)$ as follows.

Corollary 4.3. We have

$$
\gamma_{k}\left(\mathcal{P}_{r+1}\right)=\gamma_{k}\left(\mathcal{P}_{r}\right)\left[\left(1-\frac{1}{p_{r+1}}\right) \frac{G_{k}\left(p_{r+1}\right)}{G_{k}\left(p_{r}\right)}\right]
$$

where $G_{k}$ is the function given by

$$
G_{k}(x):=\sum_{i=0}^{k}\left(\sum_{S \subseteq \mathcal{P}_{\pi(x)}} \frac{L_{k-i}\left(P_{S}\right)}{\prod_{p \mid P_{S}}(p-1)}\right)\left(\begin{array}{c}
k \\
i
\end{array}\right) \gamma_{i}-\frac{1}{k+1} \sum_{S \subseteq \mathcal{P}_{\pi(x)}} \frac{L_{k+1}\left(P_{S}\right)}{\prod_{p \mid P_{S}}(p-1)}
$$

and $\pi(x)$ is the prime counting function. 
Proof. From Proposition 4.2 we have

$$
\begin{aligned}
& \gamma_{k}\left(\mathcal{P}_{r+1}\right)=\prod_{p \mid P_{r+1}}\left(1-\frac{1}{p}\right)\left(\sum_{i=0}^{k}\left(\sum_{S \subseteq \mathcal{P}_{r+1}} \frac{L_{k-i}\left(P_{S}\right)}{\prod_{p \mid P_{S}}(p-1)}\right)\left(\begin{array}{c}
k \\
i
\end{array}\right) \gamma_{i}-\frac{1}{k+1} \sum_{S \subseteq \mathcal{P}_{r+1}} \frac{L_{k+1}\left(P_{S}\right)}{\prod_{p \mid P_{S}}(p-1)}\right) \\
& =\prod_{p \mid P_{r}}\left(1-\frac{1}{p}\right)\left(1-\frac{1}{p_{r+1}}\right)\left(\sum_{i=0}^{k}\left[\sum_{S \subseteq \mathcal{P}_{r}} \frac{L_{k-i}\left(P_{S}\right)}{\prod_{p \mid P_{S}}(p-1)}+\frac{1}{p_{r+1}-1} \sum_{S \subseteq \mathcal{P}_{r}} \frac{L_{k-i}\left(p_{r+1} P_{S}\right)}{\prod_{p \mid P_{S}}(p-1)}\right]\left(\begin{array}{c}
k \\
i
\end{array}\right) \gamma_{i}\right. \\
& \left.\quad-\frac{1}{k+1} \sum_{S \subseteq \mathcal{P}_{r}} \frac{L_{k+1}\left(P_{S}\right)}{\prod_{p \mid P_{S}}(p-1)}-\frac{1}{(k+1)\left(p_{r+1}-1\right)} \sum_{S \subseteq \mathcal{P}_{r}} \frac{L_{k+1}\left(p_{r+1} P_{S}\right)}{\prod_{p \mid P_{S}}(p-1)}\right) \\
& =\gamma_{k}\left(\mathcal{P}_{r}\right)\left(\left(1-\frac{1}{p_{r+1}}\right) \frac{G_{k}\left(p_{r+1}\right)}{G_{k}\left(p_{r}\right)}\right) .
\end{aligned}
$$

The case when $k=0$, the function $A(x)$ [17, Equation 3.1] coincides with $G_{0}(x)$ and the equation 3.2 in [17] follows from the identity (21).

As a continuation, we study the behaviour of $\gamma_{k}\left(\mathcal{P}_{r}\right)$ when $k=1$ and obtain the following result.

Theorem 4.4. $\left|\gamma_{1}\left(\mathcal{P}_{r+1}\right)\right|>\left|\gamma_{1}\left(\mathcal{P}_{r}\right)\right|$ for all but finitely many $r$.

Proof. We start with the expression

$$
\begin{aligned}
G_{1}\left(p_{r}\right) & =\sum_{S \subseteq \mathcal{P}_{r}} \frac{L_{1}\left(P_{S}\right)}{\prod_{p \mid P_{S}}(p-1)} \gamma_{0}+\sum_{S \subseteq \mathcal{P}_{r}} \frac{L_{0}\left(P_{S}\right)}{\prod_{p \mid P_{S}}(p-1)} \gamma_{1}-\frac{1}{2} \sum_{S \subseteq \mathcal{P}_{r}} \frac{L_{2}\left(P_{S}\right)}{\prod_{p \mid P_{S}}(p-1)} \\
& =\gamma_{1}-\sum_{S \subseteq \mathcal{P}_{r}} \frac{\Lambda_{1}\left(P_{S}\right)}{\prod_{p \mid P_{S}}(p-1)} \gamma_{0}+\sum_{S \subseteq \mathcal{P}_{r}} \frac{\Lambda_{1}\left(P_{S}\right) \log P_{S}}{\prod_{p \mid P_{S}}(p-1)}-\frac{1}{2} \sum_{S \subseteq \mathcal{P}_{r}} \frac{\Lambda_{2}\left(P_{S}\right)}{\prod_{p \mid P_{S}}(p-1)} \\
& =\gamma_{1}-\sum_{p \in \mathcal{P}_{r}} \frac{\log p}{p-1} \gamma_{0}+\sum_{p \in \mathcal{P}_{r}} \frac{\log ^{2} p}{p-1}-\frac{1}{2} \sum_{S \subseteq \mathcal{P}_{r}} \frac{\Lambda_{2}\left(P_{S}\right)}{\prod_{p \mid P_{S}}(p-1)}
\end{aligned}
$$

The last sum can be further simplified as

$$
\begin{aligned}
\sum_{S \subseteq \mathcal{P}_{r}} \frac{\Lambda_{2}\left(P_{S}\right)}{\prod_{p \mid P_{S}}(p-1)} & =\sum_{p \in \mathcal{P}_{r}} \frac{\Lambda_{2}(p)}{p-1}+\sum_{u=1}^{r-1} \sum_{v=u+1}^{r} \frac{\Lambda_{2}\left(p_{u} p_{v}\right)}{\left(p_{u}-1\right)\left(p_{v}-1\right)} \\
& =\sum_{p \in \mathcal{P}_{r}} \frac{\Lambda_{2}(p)}{p-1}+\sum_{u=1}^{r-1} \sum_{v=u+1}^{r} \frac{\sum_{d \mid p_{u} p_{v}} \mu(d) \log ^{2}\left(\frac{p_{u} p_{v}}{d}\right)}{\left(p_{u}-1\right)\left(p_{v}-1\right)} \\
& =-\sum_{p \in \mathcal{P}_{r}} \frac{\log ^{2} p}{p-1}+2 \sum_{u=1}^{r-1} \frac{\log p_{u}}{p_{u}-1} \sum_{v=u+1}^{r} \frac{\log p_{v}}{p_{v}-1}
\end{aligned}
$$


Now by using the definition of the function $A(x)$ [17, Equation 3.1], we get

$$
G_{1}\left(p_{r+1}\right)-G_{1}\left(p_{r}\right)=\frac{\log p_{r+1}}{p_{r+1}-1}\left(\frac{3}{2} \log p_{r+1}-A\left(p_{r}\right)\right) .
$$

Now observe that

$$
\begin{aligned}
G_{1}\left(p_{r}\right) & =\gamma_{1}-\sum_{p \in \mathcal{P}_{r}} \frac{\log p}{p-1} \gamma_{0}+\frac{3}{2} \sum_{p \in \mathcal{P}_{r}} \frac{\log ^{2} p}{p-1}-\sum_{u=1}^{r-1} \frac{\log p_{u}}{p_{u}-1}\left(A\left(p_{r}\right)-A\left(p_{u}\right)\right) \\
& =\gamma_{1}-\sum_{p \in \mathcal{P}_{r}} \frac{\log p}{p-1} \gamma_{0}+\frac{3}{2} \sum_{p \in \mathcal{P}_{r}} \frac{\log ^{2} p}{p-1}-A\left(p_{r}\right) \sum_{u=1}^{r-1} \frac{\log p_{u}}{p_{u}-1}+\sum_{u=1}^{r-1} \frac{A\left(p_{u}\right) \log p_{u}}{p_{u}-1} \\
& =\gamma_{1}+\gamma_{0}^{2}+\frac{3}{2} \sum_{p \in \mathcal{P}_{r}} \frac{\log ^{2} p}{p-1}+A\left(p_{r}\right) \frac{\log p_{r}}{p_{r}-1}-A\left(p_{r}\right)^{2}+\sum_{u=1}^{r-1} \frac{A\left(p_{u}\right) \log p_{u}}{p_{u}-1} \\
& =\gamma_{1}+\gamma_{0}^{2}+\frac{3}{2} \sum_{p \in \mathcal{P}_{r}} \frac{\log ^{2} p}{p-1}+\sum_{u=1}^{r} \frac{A\left(p_{u}\right) \log p_{u}}{p_{u}-1}-A\left(p_{r}\right)^{2} .
\end{aligned}
$$

From the estimate

$$
\sum_{n \leq x} \frac{\Lambda(n)}{n}=\log x+O(1)
$$

and equation 3.7 in [17] it follows that

$$
A(x)=\log x+O(1)
$$

Therefore by the use of partial summation we get

$$
\begin{aligned}
\sum_{p \leq x} \frac{\log ^{2} p}{p-1} & =\left(A(x)-\gamma_{0}\right) \log x-\int_{1}^{x} \frac{A(t)-\gamma_{0}}{t} d t \\
& =A(x) \log x-\gamma_{0} \log x-\int_{1}^{x} \frac{\log t}{t} d t+O\left(\int_{1}^{x} \frac{1}{t} d t\right) \\
& =\frac{\log ^{2} x}{2}+O(\log x) .
\end{aligned}
$$

Similarly one can deduce that

$$
\sum_{u=1}^{r} \frac{A\left(p_{u}\right) \log p_{u}}{p_{u}-1}=\sum_{u=1}^{r} \frac{\log ^{2} p_{u}}{p_{u}-1}+O\left(\log p_{r}\right)
$$

and

$$
A\left(p_{r}\right)^{2}=\log ^{2} p_{r}+O\left(\log p_{r}\right) .
$$


Using the above estimates we get

$$
\frac{G_{1}\left(p_{r}\right)}{p_{r+1}}=\frac{1}{p_{r+1}}\left(\frac{\log ^{2} p_{r}}{4}+O\left(\log p_{r}\right)\right)
$$

and for $r$ increasing arbitrarily large we have

$$
\begin{aligned}
G_{1}\left(p_{r+1}\right)-G_{1}\left(p_{r}\right) & =\frac{\log p_{r+1}}{p_{r+1}-1}\left(\frac{3}{2} \log p_{r+1}-A\left(p_{r}\right)\right) \\
& =\frac{1}{p_{r+1}-1}\left(\frac{3}{2} \log ^{2} p_{r+1}-\log p_{r} \log p_{r+1}+O\left(\log p_{r+1}\right)\right) \\
& >\frac{1}{p_{r+1}}\left(\frac{1}{2} \log ^{2} p_{r+1}+O\left(\log p_{r+1}\right)\right) \\
& >\frac{G_{1}\left(p_{r+1}\right)}{p_{r+1}} .
\end{aligned}
$$

Hence we get the desired inequality.

Values of $\gamma_{k}\left(\mathcal{P}_{r}\right)$ for a few initial values of $r$ and $k$ are given in Table 1.

\subsection{A closed form expression for the first and second generalized Stieltjes con- stant}

We conclude this section with an another important generalization of Euler constant $\gamma$ arising from the study of Laurent series expansion of Hurwitz zeta function $\zeta(s, x)$ around the point $s=1$. In particular the expansion is given by

$$
\zeta(s, a)=\frac{1}{s-1}+\sum_{n=0}^{\infty} \frac{(-1)^{n} \gamma_{n}(a)}{n !}(s-1)^{n}
$$

where the constants $\gamma_{n}(a)$ (known as Generalized Stieltjes constants) was shown by Berndt [4, Theorem 1] to have the following asymptotic representation

$$
\gamma_{n}(a)=\lim _{N \rightarrow \infty}\left(\sum_{k=0}^{N} \frac{\log ^{n}(k+a)}{k+a}-\frac{\log ^{n+1}(N+a)}{n+1}\right) .
$$

Here we can see that when $a=1,(22)$ reduces to (1). For a good account of history and survey of results related to the constants $\gamma_{n}(a)$ see [6]. As mentioned by Blagouchine that these constants are much less studied than the Euler Stieltjes constants $\gamma_{n}$. It was conjectured in 2015 by Blagouchine [5, p. 103] that "any generalized Stieltjes constant of the form $\gamma_{1}(r / q)$, where $r$ and $q$ are positive integers such that $r<q$, may be expressed by means of the Euler's constant $\gamma$, the first Stieltjes constant $\gamma_{1}$, the logarithm of the $\Gamma$ function at rational argument(s) and some relatively simple, perhaps elementary, function." Using a large number of calculations involving Malmsten's integrals, this conjecture was 
settled by Blagouchine himself in 2015 by proving [6, Theorem 1] four equivalent closed form expressions [6, Eq. 37,50,53,55] for the first generalized Stieltjes constant $\gamma_{1}(r / q)$. In particular equation 50 in [6] is the following identity.

$$
\begin{aligned}
\gamma_{1}\left(\frac{r}{q}\right)= & \gamma_{1}-\gamma \log 2 q-\frac{\pi}{2}(\gamma+\log 2 \pi q) \cot \left(\frac{\pi r}{q}\right)+\sum_{l=1}^{q-1} \cos \frac{2 \pi r l}{q} \cdot \zeta^{\prime \prime}\left(0, \frac{l}{q}\right) \\
& +\pi \sum_{l=1}^{q-1} \sin \frac{2 \pi r l}{q} \cdot \log \Gamma\left(\frac{l}{q}\right)+(\gamma+\log 2 \pi q) \sum_{l=1}^{q-1} \cos \frac{2 \pi r l}{q} \cdot \log \sin \left(\frac{\pi l}{q}\right) \\
& -\log ^{2} 2-\log 2 \cdot \log \pi q-\frac{1}{2} \log ^{2} q .
\end{aligned}
$$

The identity (23) was recently proved by Coffey [15, Proposition 1] using functional equation of Hurwitz zeta function. We give here a much simpler proof with the help of an identity of Shirasaka.

\section{Proof of (23)}

Using Laurent series expansion (7) of $\zeta(s ; r, q)$ Shirasaka proved that [53, Theorem (ii)] the constants $\gamma_{k}(r, q)$ are related with generalized Stieltjes constants by the following identity:

$$
\gamma_{k}(r, q)=\frac{1}{q} \sum_{j=0}^{k}\left(\begin{array}{l}
k \\
j
\end{array}\right)(\log q)^{k-j} \gamma_{j}\left(\frac{r}{q}\right)-\frac{\log ^{k+1} q}{q(k+1)} .
$$

By the use of the work of Deninger [16], Kanemitsu gave the closed form expressions for $\gamma_{0}(r, q)$ [30, Eq. 3.7], $\gamma_{1}(r, q)$ [30, Eq. 3.10] and $\gamma_{2}(r, q)$ [30, Eq. 3.11] in terms of Deninger function $R_{k}(x)$ which satisfies [30, Eq. 2.7]

$$
R_{k}(x)=(-1)^{k+1} \frac{\partial^{k}}{\partial s^{k}} \zeta(0, x) .
$$

These expressions for $\gamma_{0}(r, q), \gamma_{1}(r, q), \gamma_{2}(r, q)$ and the identity (24) readily gives the desired expression for $\gamma_{j}\left(\frac{r}{q}\right)$ with $j=0,1,2$.

\section{Concluding remarks}

Just like, $\tilde{\Delta}_{2}(x)$ a more general $\tilde{\Delta}_{k}(x)$ is defined as the summatory function of all those natural number $n$ which can be written as the product of $k$ many factors with each factor of the form $n_{i} \equiv r_{i} \bmod q_{i}$ for $i=1,2, \ldots, k$. Any interested reader can derive results for $\tilde{\Delta_{k}}(x)$ parallel to Theorem 2.7 using the results of [25] and [54]. However the expressions become more complicated and tedious to handle. On the other hand compared to the case of $\gamma(r, q)$ the arithmetic nature of $\zeta_{k}(\alpha, r, q)$ seems to be even harder to investigate because 
Baker's theory on linear forms of logarithm of algebraic numbers seems to be of no help. Nevertheless we hope to see some progress in the understanding of analytic and arithmetic nature of these constants in future.

\section{Acknowledgements}

The authors would like to thank the referee for some highly useful suggestions which improved the quality of this paper. The first author thanks NBHM for providing partial support for this work. The second author thanks CSIR for financial support. 


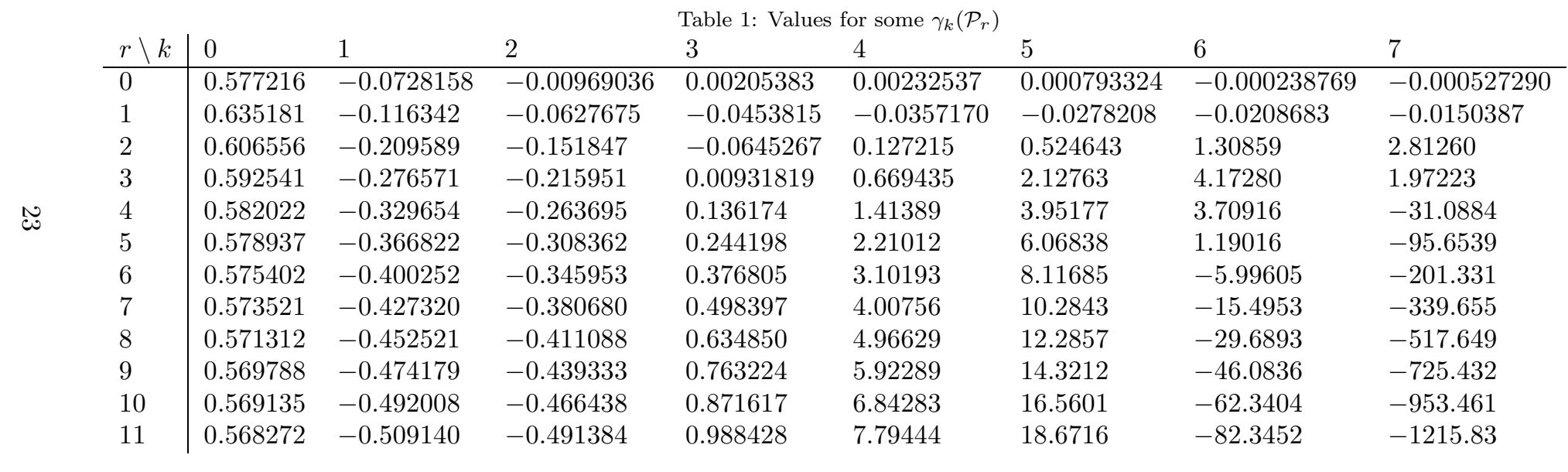




\section{References}

[1] Dorin Andrica and László Tóth, Some remarks on Stieltjes constants of the zeta function, Stud. Cerc. Mat. 43 (1991), no. 1-2, 3-9. MR 1138694

[2] Tom M. Apostol, Introduction to analytic number theory, Springer-Verlag, New York-Heidelberg, 1976, Undergraduate Texts in Mathematics. MR 0434929

[3] R. Balasubramanian and V. Kumar Murty, Zeros of Dirichlet L-functions, Ann. Sci. École Norm. Sup. (4) 25 (1992), no. 5, 567-615. MR 1191737

[4] Bruce C. Berndt, On the hurwitz zeta-function, Rocky Mountain J. Math. 2 (1972), no. 1, 151-158.

[5] Iaroslav V. Blagouchine, Rediscovery of Malmsten's integrals, their evaluation by contour integration methods and some related results, Ramanujan J. 35 (2014), no. 1, 21-110. MR 3258600

[6] _ A theorem for the closed-form evaluation of the first generalized Stieltjes constant at rational arguments and some related summations, J. Number Theory 148 (2015), 537-592. MR 3283193

[7] Khristo N. Boyadzhiev, On Taylor's coefficients of the Hurwitz zeta function, JP J. Algebra Number Theory Appl. 12 (2008), no. 1, 103-112. MR 2494076

[8] W. E. Briggs, Some constants associated with the Riemann zeta-function, Michigan Math. J. 3 (1955-56), 117-121. MR 0076858

[9] $\quad$ The irrationality of $\gamma$ or of sets of similar constants, Norske Vid. Selsk. Forh. (Trondheim) 34 (1961), 25-28. MR 0139579

[10] W. E. Briggs and S. Chowla, The power series coefficients of $\zeta(s)$, Amer. Math. Monthly 62 (1955), 323-325. MR 0069209

[11] H. M. Bui and Micah B. Milinovich, Central values of derivatives of Dirichlet L-functions, Int. J. Number Theory 7 (2011), no. 2, 371-388. MR 2782664

[12] T. Chatterjee and S. Gun, The digamma function, Euler-Lehmer constants and their p-adic counterparts, Acta Arith. 162 (2014), no. 2, 197-208. MR 3167891

[13] Mark W. Coffey, New results on the Stieltjes constants: asymptotic and exact evaluation, J. Math. Anal. Appl. 317 (2006), no. 2, 603-612. MR 2209581

[14] _ Series representations for the Stieltjes constants, Rocky Mountain J. Math. 44 (2014), no. 2, 443-477. MR 3240509 
[15] _ Functional equations for the Stieltjes constants, Ramanujan J. 39 (2016), no. 3, 577-601. MR 3472126

[16] Christopher Deninger, On the analogue of the formula of Chowla and Selberg for real quadratic fields, J. Reine Angew. Math. 351 (1984), 171-191. MR 749681

[17] H.G. Diamond and K. Ford, Generalized Euler constants, Mathematical Proceedings of the Cambridge Philosophical Society 145 (2008), no. 1, 2741.

[18] Jack Diamond, The p-adic log gamma function and p-adic Euler constants, Trans. Amer. Math. Soc. 233 (1977), 321-337. MR 0498503

[19] Karl Dilcher, Generalized Euler constants for Arithmetical Progressions, Mathematics of Computation 59 (1992), no. 199, 259-282.

[20] J. Furuya and Y. Tanigawa, Explicit representations of the integral containing the error term in the divisor problem, Acta Math. Hungar. 129 (2010), no. 1-2, 24-46. MR 2725833

[21] Sanoli Gun, V. Kumar Murty, and Ekata Saha, Linear and algebraic independence of generalized Euler-Briggs constants, Journal of Number Theory 166 (2016), no. Supplement C, 117 - 136.

[22] Sanoli Gun, Ekata Saha, and Sneh Bala Sinha, Transcendence of generalized Euler-Lehmer constants, J. Number Theory 145 (2014), 329-339. MR 3253307

[23] Max Gut, Die Zetafunktion, die Klassenzahl und die Kronecker'sche Grenzformel eines beliebigen Kreiskörpers, Comment. Math. Helv. 1 (1929), no. 1, 160-226. MR 1509394

[24] M. N. Huxley, Exponential sums and lattice points. III, Proc. London Math. Soc. (3) 87 (2003), no. 3, 591-609. MR 2005876

[25] Makoto Ishibashi and Shigeru Kanemitsu, Dirichlet series with periodic coefficients, Results Math. 35 (1999), no. 1-2, 70-88. MR 1678080

[26] Hideaki Ishikawa, On the coefficients of the Taylor expansion of the Dirichlet L-function at $s=1$, Acta Arith. 97 (2001), no. 1, 41-52. MR 1819621

[27] Aleksandar Ivić, The Riemann zeta-function, A Wiley-Interscience Publication, John Wiley \& Sons, Inc., New York, 1985, The theory of the Riemann zeta-function with applications. MR 792089

[28] Aleksandar Ivić, On the Laurent Coefficients of Certain Dirichlet Series, Publications de l'Institut Mathmatique 53(67) (1993), no. 73, 23-36 (eng). 
[29] H. Iwaniec and P. Sarnak, Dirichlet L-functions at the central point, Number theory in progress, Vol. 2 (Zakopane-Kościelisko, 1997), de Gruyter, Berlin, 1999, pp. 941-952. MR 1689553

[30] S. Kanemitsu, On evaluation of certain limits in closed form, Théorie des nombres (Quebec, PQ, 1987), de Gruyter, Berlin, 1989, pp. 459-474. MR 1024583

[31] Charles Knessl and Mark W. Coffey, An effective asymptotic formula for the Stieltjes constants, Math. Comp. 80 (2011), no. 273, 379-386. MR 2728984

[32] J. Knopfmacher, Generalised euler constants, Proceedings of the Edinburgh Mathematical Society 21 (1978), no. 1, 2532.

[33] Jeffrey C. Lagarias, Euler's constant: Euler's work and modern developments, Bulletin of the American Mathematical Society 50 (2013), no. 4, $527-628$.

[34] A. F. Lavrik, M. I. Israilov, and Ž. Ëdgorov, Integrals containing the remainder term of the divisor problem, Acta Arith. 37 (1980), 381-389. MR 598891

[35] D. H. Lehmer, Euler constants for arithmetical progressions, Acta Arith. 27 (1975), 125-142. MR 0369233

[36] - The sum of like powers of the zeros of the Riemann zeta function, Math. Comp. 50 (1988), no. 181, 265-273. MR 917834

[37] Jinjiang Li and Min Zhang, On the fourth power moment of the error term for the divisor problem with congruence conditions, Int. J. Number Theory 14 (2018), no. 6, 1525-1546. MR 3827944

[38] J. J. Y. Liang and John Todd, The Stieltjes constants, J. Res. Nat. Bur. Standards Sect. B 76B (1972), 161-178. MR 0326974

[39] Kui Liu, On higher-power moments of the error term for the divisor problem with congruence conditions, Monatsh. Math. 163 (2011), no. 2, 175-195. MR 2794196

[40] Philippe Michel and Jeffrey VanderKam, Non-vanishing of high derivatives of Dirichlet L-functions at the central point, J. Number Theory 81 (2000), no. 1, 130-148. MR 1743500

[41] Dragiša Mitrović, The signs of some constants associated with the Riemann zeta-function, Michigan Math. J. 9 (1962), 395-397. MR 0164941

[42] Wolfgang Müller and Werner Georg Nowak, Third power moments of the error terms corresponding to certain arithmetic functions, Manuscripta Math. 87 (1995), no. 4, 459-480. MR 1344601 
[43] M. Ram Murty and N. Saradha, Euler-Lehmer constants and a conjecture of Erdos, Journal of Number Theory 130 (2010), no. 12, 2671 - 2682.

[44] M. Ram Murty and Naomi Tanabe, On the nature of $e^{\gamma}$ and non-vanishing of derivatives of $L$-series at $s=1 / 2$, J. Number Theory 161 (2016), 444456. MR 3435736

[45] M.R. Murty and A. Zaytseva, Transcendence of generalized Euler constants, American Mathematical Monthly 120 (2013), no. 1, 48-54, cited By 4.

[46] W. G. Nowak, On the Piltz divisor problem with congruence conditions. II, Abh. Math. Sem. Univ. Hamburg 60 (1990), 153-163. MR 1087125

[47] Werner Georg Nowak, On a divisor problem in arithmetic progressions, J. Number Theory 31 (1989), no. 2, 174-182. MR 987570

[48] S. Ramanujan, A series for Euler's constant $\gamma$, Messenger of Mathematics 46 (1917), 73-80.

[49] Don Redmond, A generalization of a theorem of Ayoub and Chowla, Proc. Amer. Math. Soc. 86 (1982), no. 4, 574-580. MR 674083

[50] Corrections and additions to: "A generalization of a theorem of Ayoub and Chowla", Proc. Amer. Math. Soc. 90 (1984), no. 2, 345-346. MR 727263

[51] Hans-Egon Richert, Ein Gitterpunktproblem, Math. Ann. 125 (1953), 467471. MR 0054655

[52] Sumaia Saad Eddin, Explicit upper bounds for the Stieltjes constants, J. Number Theory 133 (2013), no. 3, 1027-1044. MR 2997785

[53] S. Shirasaka, On the Laurent coefficients of a class of Dirichlet series, Results Math. 42 (2002), no. 1-2, 128-138. MR 1934231

[54] R. Sitaramachandra Rao, An integral involving the remainder term in the Piltz divisor problem, Acta Arith. 48 (1987), no. 1, 89-92. MR 893465

[55] K. Soundararajan, Nonvanishing of quadratic Dirichlet L-functions at $s=$ $\frac{1}{2}$, Ann. of Math. (2) 152 (2000), no. 2, 447-488. MR 1804529

[56] E. C. Titchmarsh, The theory of the Riemann zeta-function, second ed., The Clarendon Press, Oxford University Press, New York, 1986, Edited and with a preface by D. R. Heath-Brown. MR 882550

[57] Masao Toyoizumi, On the size of $L^{(k)}(1, \chi)$, J. Indian Math. Soc. (N.S.) 60 (1994), no. 1-4, 145-149. MR 1292132

[58] Nan Yue Zhang and Kenneth S. Williams, Some results on the generalized Stieltjes constants, Analysis 14 (1994), no. 2-3, 147-162. MR 1302533 